\title{
The Neurocognition of Translation and Interpreting
}

GarcíA, AdOLFo M. 2019. The Neurocognition of Translation and Interpreting. Amsterdam/Philadelphia: John Benjamins Publishing Company.

Reviewed by OBED EBENEZER $\mathrm{S}$

The advent of the 'Cognitive Turn' in Translation Studies has resulted in shifting the focus away from the text, culture, and the reader (and the multiplicity of issues arising out of such concerns), to the translator, effecting what Halverson terms as the 'Centrality of the Translator' (2014:116). Till the late 1900 s, translation was viewed purely as an exercise between languages. Later, issues such as the selection of the source text and the target language, the reception of the translated text, the influence of ideology and power relations etc. came to the fore, influenced by perspectives from disciplines such as Cultural Studies, Gender Studies, and Sociology. With the development of Cognitive Science and its foray into Artificial Intelligence, the interest shifted to understanding the human mind and the various functions and processes that take place inside the brain. Even so, perspectives on human cognition have tended towards the view that cognition is situated, embodied, and extended, thereby looking at contextual and environmental factors that influence the translator. However, in this volume, the focus of the author moves to the very centre of the 'centrality of the translator' approach, that is, the brain of the translator.

Alves \& Albir (2017: 537) state that research on the cognitive aspects of translation has 'focused primarily on three interrelated fields, namely, the translation process, translation competence, and the acquisition of translation competence'. Translation process research (TPR), which deals with how the process of translation is carried out by the translator, was found to be an area that had received the most attention in the 
discipline of Translation Studies since 2010 (Li \& Lei 2019: v). This highlights not only the increasing interest in this topic, but also the possibilities that this interface of disciplines holds. Indeed, the present volume also deals with TPR, but goes a step further in presenting scientific studies that explore the changes that take place in the brain during the translation process.

Because the discipline of Translation Studies had long been grounded in Humanities, and primarily handled by language and/or literary practitioners, a rigorous scientific approach towards translation had been missing (or viewed with suspicion) till the recent past. Even though the interest in human cognition arose in the late 1980s, such studies, though empirical, relied mostly on observational and behavioural data. The recent advances in scientific and medical technology have made it possible to observe what occurs inside the brain during different activities using tools such as Functional Magnetic Resonance Imaging (FMRI), electroencephalograph (EEG), functional near-infrared spectroscopy (FNIRS) etc. This has opened up a vast area of hitherto unexplored avenue of interdisciplinary approaches to both understanding what takes place inside the brain during translation, and also to closely observe the effects of such changes. The Neurocognition of Translation and Interpreting not only brings together the various methods, studies and findings that delve into what actually happens inside the human brain during the process of translation/interpretation, but also provides a more scientifically grounded platform for further research in this field.

García admits that neuroscientific research is far from being perfect, but also hastens to add that this lack does not nullify the contributions made towards the understanding of the brain, but rather serves to enhance our present (and limited) 
knowledge. Within the span of eight chapters, the author manages to introduce the basic concepts of neuroscience to the uninitiated beginner, describe the methodological process and tools used, summarize the major findings on which the present-day investigations are founded upon, and then proceeds to examine the anatomy and functions of the human brain including the cortical and subcortical regions, hemispheres, and the electrophysiological variations which influence the verbal and non-verbal information processing as well as behaviour. Towards the end, the evidence that the training and development of interpreting skills results in anatomical and functional enhancement in the brain, is put forward. The volume also asserts that neurocognitive research holds great potential for Translation and Interpretation Studies.

The author also puts it in no uncertain terms that the scientific evidence presented in this volume is specifically based on interlingual translation, and as the functioning of the brain differs for each process, its findings cannot be assumed to apply to other types of translation (such as intralingual or intersemiotic). At the same time, he also states that the evidences laid forth are only the results of empirical studies and probable inferences, and are not infallible.

The volume opens by giving a brief overview about the various non-neural cognitive approaches, and categorises them as rationalist, observational, introspective, corpus-based, and quantitative. Then, the different ways in which a neurological approach can contribute towards our understanding of cognitive processes are discussed, including impairment of skills, the insight into the inner duration of unobservable processes, the interaction between different neural systems, functional neurological differences in translation competence and expertise as opposed to untrained translation, and the ability to stimulate specific parts of the brain allow for 
controlled manipulation to obtain causal evidence for associative patterns. A short history and the various milestones in the neurocognitive research of translation are also laid down.

The various methods and tools used for brain research with respect to Translation Studies are listed out and described in the chapter aptly titled as 'The toolkit'. Relevant studies, which taken together can provide a substantial framework for research, are classified as single-case where individuals are studied; single-group where multiple participants take up the same tests; between-group where groups differing by a single variable are analysed; and pre/post-studies where groups are assessed before and after a particular training has been imparted. Nueroscientific methods are of two types: noninvasive, such as FMRI, PET imaging, and EEG which analyse brain activity from outside; and invasive, such as direct electro-stimulation and intracranial EEG where tools are physically inserted inside the brain to study it.

The findings from various studies compiled and presented in this book provide insights into how mental processes vary during translation in terms of directionality, the function of unit-specific variables, and the nature of competence and expertise. Translation, as opposed to mono-lingual reading, involves activation of different frontostriatal and temporal regions. The direction of translation causes modulations in the cortical and subcortical hubs. Frontostriatal and temporoparietal regions are distinctly specialized for processing different word types such as action verbs and concrete nouns respectively. Of significant interest is the evidence that the brain of translators who engage in simultaneous interpretation undergo structural and functional changes with regard to linguistic and executive roles, resulting in superior mental coordination skills. 
Cognitive approaches have enabled a deeper understanding of the process of translation which hitherto could not have been imagined using other approaches. It also highlights the importance and need for fostering research across disciplines, as the human mind is too complex and heterogeneous to be viewed from a single perspective. However, the current research is not without fault- low sample sizes result in lesser reliability, only a handful of variables has been described and controlled, most studies have focussed on translation only at the word and sentence level, and the statistical criteria has not been standardized across tests.

The volume ends with a series of questions and answers that serve to sum up the findings discussed in the previous chapters. Adolfo M. García concludes by suggesting that the final words in this book should not be read as 'The End', but rather as 'To be Continued', thus hoping that it would inspire further work to be taken up on this topic. That this review has been written only serves as a witness to the fact that this wish has come to fruition.

\section{References}

Alves, Fabio, AND Amparo Hurtado Albir. 2017. Evolution, Challenges, and Perspectives for Research on Cognitive Aspects of Translation. In Schwieter, John W \& Aline Ferreira (eds.), The Handbook of Translation and Cognition. 537-554. Hoboken, NJ: John Wiley \& Sons, Inc.

García, Adolfo M. 2019. The Neurocognition of Translation and Interpreting. Amsterdam / Philadelphia: John Benjamins Publishing Company.

HALVERSON, SANDRA L. 2014. Reorienting Translation Studies: Cognitive Approaches and the Centrality of the Translator. In Juliane House (ed.), Translation: A 
Obed Ebenezer S

Multidisciplinary Approach. 116-139. Hampshire/New York: Palgrave Macmillan.

Li, Defeng, and Victoria LAI Cheng Lei. 2019. Introduction. In Defeng Li, Victoria Lai Cheng Lei, and Yuanjian He (eds.), Researching Cognitive Processes of Translation. V-x. Singapore: Springer Nature Singapore Pte Ltd.

$$
* * *
$$

Cite this work:

EBENEZER, OBED S. 2020. The Neurocognition of Translation and Interpreting. Review of The Neurocognition of Translation and Interpreting, by Adolfo M. García. Translation Today, Vol. 14(1). 129-134. DOI: $10.46623 / \mathrm{tt} / 2020.14 .1 . b r 2$ 